\title{
Editorial: A Message from the Editor-in-Chief
}

Dear ACM TACO community,

I am honored to serve as the next Editor-in-Chief of ACM Transactions on Architecture and Code Optimization. TACO is the leading journal focused on computer architecture and code optimization. It is an exciting time in our field, especially given the number of advances in open-source architectures, accelerator architectures, hardware security, domain-specific languages, emerging memory technologies, and runtime systems. Given TACO's journal-first model, this quality publication continues to break new ground in research publications.

My vision for the journal is to build on a number of best practices already in place with TACO, which include: short (two-month) decisions on submissions, collaboration with HiPEAC (which began in 2010 and increased submissions significantly), and maintaining a strong Distinguished Reviewer Board. My plans for changes and enhancements to TACO include: (1) increasing the diversity of our editorial board, (2) establishing ties to additional computer architecture and compiler conferences, and (3) increasing the impact factor of the journal.

I am pleased to announce the addition of the following Associate Editors:

- Ramon Canal, Universitat Politecnica de Catalunya

- Yunsi Fei, Northeastern University

- Russ Joseph, Northwestern University

- Hyesoon Kim, Georgia Tech

- Chia-Lin Yang, National Taiwan University

- Antonia Zhai, University of Minnesota

We would like to thank the following Associate Editors who are completing their terms on the Editorial Board:

- Koen Bertels, Delft University

- Martha Kim, Columbia University

We will be developing a policy on ethics and professional conduct specific for ACM TACO, that builds on ACM's current policies, though focuses on specific issues related to publications and reviewing. We feel this will help to provide a more inclusive environment for this journal.

The ongoing collaboration between HiPEAC and ACM TACO has demonstrated the many benefits of the journal-first model. I have served as both general chair and program chair of HiPEAC and fully appreciate the importance of this continued collaboration. One initiative I plan to pursue is to explore building relationships with other leading conferences in our field. I believe this initiative is timely, given how many conferences over the past year have moved to a virtual format, and given how this collaboration has benefitted TACO.

\footnotetext{
ACM Reference format: 16 (August 2020), 2 pages. https://doi.org/10.1145/3409369

(C) 2020 Copyright held by the owner/author(s).

1544-3566/2020/08-ART16

https://doi.org/10.1145/3409369
}

David Kaeli. 2020. Editorial: A Message from the Editor-in-Chief. ACM Trans. Archit. Code Optim. 17, 3, Article 
Finally, to increase the impact factor of ACM TACO, we will need to increase readership and the number of high-quality submissions. Collaboration with additional conferences should have a positive influence on the impact factor. In addition, we will be adopting awards recognizing the top papers appearing in TACO each year. This should help to raise the interest and prestige of the journal. ACM TACO moved to Gold Open Access in 2018 for a period of four years. We will be accessing the impact of this change over the next 2 years, evaluating its impact on increased readership and citation counts. We will also leverage social media to advertise each issue of ACM TACO, as well as continue to build out the ACM TACO website: https://dl.acm.org/journal/.

Finally, I would like to recognize the outgoing Editor-in-Chief, Koen De Bosschere from Ghent University. Koen has been a thought-leader for the journal and developed a number of innovative best practices that have helped this journal grow in both submissions and prestige. I am pleased to announce that Koen will continue to serve in the role of TACO's Administrator. We thank Koen for his continued outstanding service and contributions.

I look forward to working with the Editorial Board and the ACM Publications team to continue to strengthen this journal. If you have suggestions on how to improve ACM TACO, please feel free to contact me.

Dave Kaeli ACM TACO Editor-in-Chief 